\title{
Uniqueness, continuity and the existence of implicit functions in constructive analysis
}

\author{
H. Diener and P. Schuster
}

\begin{abstract}
We extract a quantitative variant of uniqueness from the usual hypotheses of the implicit function theorem. Not only does this lead to an a priori proof of continuity, but also to an alternative, full proof of the implicit function theorem. Additionally, we investigate implicit functions as a case of the unique existence paradigm with parameters.
\end{abstract}

\section{Introduction}

To prove the differentiability of an implicit function one often relies on its continuity. The latter is mostly seen as a by-product of the common construction of the implicit function as the limit of a uniformly convergent sequence of continuous functions. We now show that the continuity of the implicit function is prior to its existence, and thus is independent of any particular construction. More specifically, we deduce the continuity of the implicit function from a quantitative strengthening of the uniqueness, which in turn follows from the hypotheses one needs to impose on the equation that the implicit function is expected to satisfy. The same quantitative strengthening of uniqueness enables us to give an alternative existence proof for implicit functions that is fully constructive in Bishop's sense. To summarise this, we will prove constructively.

Theorem. Let $U \subseteq \mathbb{R}^{n}$ and $V \subseteq \mathbb{R}^{m}$ be open neighbourhoods of $a \in \mathbb{R}^{n}$ and $b \in \mathbb{R}^{m}$, respectively, with $m, n \geqslant 1$ and let $F: U \times V \rightarrow \mathbb{R}^{m}$ be a continuously differentiable function such that $(\partial F / \partial y)(a, b)$ is invertible. Then there exist compact neighbourhoods $U_{\lambda}^{0} \subseteq U$ and $V_{\lambda}^{0} \subseteq V$ of $a$ and $b$, respectively, and a function $f: U_{\lambda}^{0} \rightarrow V_{\lambda}^{0}$ such that $F(x, f(x))=0$ for every $x \in U_{\lambda}^{0}$; moreover, this function is necessarily continuous.

We use ideas from [6], where this theorem has been proved in the case $m=1$. That existence proof relies on monotonicity and therefore cannot be carried over to the general case of $m>1$, where we need to employ an extreme value argument. Similar considerations in related contexts can be found in [11]: in $\S 3.3$ during the proof of the implicit function theorem via the inverse mapping theorem and in the proof of Banach's fixed point theorem in $\S 3.4$. We refer to [12, 21] for the implicit function theorem and the open mapping theorem in computable analysis à la Weihrauch $[\mathbf{2 0}]$.

The predecessor [18] of this paper essentially contains the same material as far as uniqueness and continuity of the implicit function are concerned. However, when it comes to proving existence, $[\mathbf{1 8}]$ uses the intrinsically non-constructive argument that a continuous function on a compact set attains its minimum. This argument fails constructively, unless one has that there is quantitatively at most one point at which the minimum can be attained. In fact, there is a heuristic principle valid [19] even in Bishop-style constructive mathematics without countable choice: if a continuous function on a complete metric space has approximate roots

Received 28 January 2010; revised 15 July 2010.

2000 Mathematics Subject Classification 03F60 (primary), 26E40, 03F55 (secondary).

This article is an amended version of a conference proceedings paper of the same title [9]. 
and in a quantitative manner at most one root, then it actually has a root. We refer to $[\mathbf{1 7}, \mathbf{1 9}]$ for the rich history of this principle and the concepts involved therein.

In the case of implicit functions, the required additional hypothesis is contained in the quantitative variant of uniqueness which follows from the hypothesis of the implicit function theorem. Therefore, in order to prove the implicit function theorem we need only prove that for each parameter the given equation admits approximate solutions. Altogether we achieve the existence of an exact solution at every parameter and then, by the principle of unique choice, the existence of an implicit function as the one and only function which assigns to every parameter the solution uniquely determined by this parameter.

The present paper as a whole is conceived in the realm of Bishop's constructive mathematics $[4,5,7,8]$. Compared with the - so-called classical - customary way of doing mathematics, the principal characteristic of the framework created by Bishop is the exclusive use of intuitionistic logic, which allows one to view Bishop's setting as a generalisation of classical mathematics [14]. Moreover, we follow [15] in performing constructive mathematics à la Bishop without countable choice; this requires us to understand real numbers as located Dedekind cuts. In particular, the so-called cotransitivity property 'if $x<y$, then $x<z$ or $z<y$ ' amounts to saying that the Dedekind cut $z$ is located whenever $x, y$ are rational numbers, and follows by approximation in the general case.

Avoiding countable choice is further indispensable, because we want our work to be expressible in constructive Zermelo-Fraenkel set theory (CZF) as begun in [1]: countable choice is independent of CZF. Details on this and on CZF in general can be found in $[2,13]$. We will, however, use the principle of unique choice, sometimes called the principle of non-choice. By the functions-as-graphs paradigm common to set theory, unique choice is trivially in CZF.

\section{Preliminaries}

We recall that in Bishop's setting every differentiable function comes with a continuous derivative [5, Chapter 2, Section 5]. In other words, for Bishop every differentiable function is by definition continuously differentiable. We nonetheless keep speaking of continuously differentiable functions in order to facilitate any reading by a classically trained person. Note in this context that in Bishop's framework, continuity means uniform continuity on every compact (that is, totally bounded and complete) subset of the domain; see [16] for a discussion of this. A function with values in $\mathbb{R}^{n}$ is differentiable, if every component function is.

Although in the work of Bishop and of his followers there is barely any talk of (partial or total) differentiability for functions of several real variables, we do not develop this concept in the present paper: we believe that the task of checking the classical route as far as necessary can indeed be performed in a relatively straightforward way, and is sometimes simplified by Bishop's assumption of the automatic continuity of the derivative.

If not mentioned otherwise, $\|\cdot\|$ denotes the Euclidean norm.

Next, because of a lack of appropriate references in the constructive literature, we transfer two facts from real analysis. With [5, Chapter 2, Theorems 5.4 and 6.8] at hand the standard proofs indeed go through constructively. (For instance, the proofs of Satz 5 and of its corollary given in [10, I, Section 6] require only one addendum to the proof of the Hilfssatz: for all $K, L \in \mathbb{R}$ with $L \geqslant 0$ the implication 'if $K^{2} \leqslant K L$, then $K \leqslant L$ ' is also valid constructively. To verify this, assume that $K^{2} \leqslant K L$; it suffices to prove that $K<L+\varepsilon$ for every $\varepsilon>0$. For each $\varepsilon>0$ either $0<K$ or $K<\varepsilon$. In the former case, multiplying $K^{2} \leqslant K L$ by $1 / K>0$ yields $K \leqslant L$; in the latter case we have $K<L+\varepsilon$ because $L \geqslant 0$.)

Lemma 2.1. Let $g: W \rightarrow \mathbb{R}^{n}$ be a continuously differentiable mapping on an open set $W \subseteq \mathbb{R}^{m}$, and $c, d \in W$. If the line segment between $c$ and $d$ lies entirely in $W$, then

$$
g(d)-g(c)=\left(\int_{0}^{1} D g(c+t(d-c)) d t\right) \cdot(d-c) .
$$


COROLlary 2.2. Under the hypotheses of Lemma 2.1 we have

$$
\|g(d)-g(c)\| \leqslant \sup _{t \in[0,1]}\|D g(c+t(d-c))\| \cdot\|d-c\| .
$$

While [5, Chapter 2, Lemma 5.5] is an approximative version of Rolle's theorem, our next lemma is a variant of the contrapositive.

Lemma 2.3. Let $c<d$, let $h:[c, d] \rightarrow \mathbb{R}$ be a continuously differentiable function and suppose that there is $r>0$ such that $h^{\prime}(x)>r$ for all $x \in[c, d]$. Then $h(c)<h(d)$.

Proof. Assume that $h(d)-h(c)<r(d-c) / 4$. By the mean value theorem [5, Theorem 5.6] there exists $\xi \in[c, d]$ such that

$$
\left|h^{\prime}(\xi)(d-c)-(h(d)-h(c))\right|<\frac{r(d-c)}{2} .
$$

Then

$$
\begin{aligned}
h^{\prime}(\xi)(d-c)-(h(d)-h(c)) & >r(d-c)-(h(d)-h(c)) \\
& >r(d-c)-\frac{r(d-c)}{4} \\
& >\frac{r(d-c)}{2} .
\end{aligned}
$$

Hence we get a contradiction, and thus $h(d)-h(c)>0$.

The last lemma in this section is an approximative substitute for the classical result that if the minimum of a differentiable function on a compact set is attained at a point in the interior, then the gradient of that function vanishes at this point. (This implication would also hold in constructive analysis where, however, one cannot expect to find such a point at all; see for example $[\mathbf{1 7}, \mathbf{1 9}]$.

Lemma 2.4. Let $W \subseteq \mathbb{R}^{n}$ be an open neighbourhood of $[0,1]^{n}$ and let $h: W \rightarrow \mathbb{R}$ be a continuously differentiable function. If there is a point $\xi \in[0,1]^{n}$ and $s>0$ such that

$$
h(x)>h(\xi)+s
$$

for all $x \in \partial[0,1]^{n}$, then for every $\varepsilon>0$ there exists $y \in[0,1]^{n}$ such that $\|\nabla h(y)\|<\varepsilon$.

Proof. For convenience we will use the supremum norm on $\mathbb{R}^{n}$ throughout this proof. Choose $N \in \mathbb{N}$ such that for all $x, y \in[0,1]^{n}$, if $\|y-x\|<2^{-N}$ then both

$$
\|\nabla h(x)-\nabla h(y)\|<\frac{\varepsilon}{4}
$$

and

$$
|h(x)-h(y)|<s
$$

Let

$$
G=\left\{\left(\frac{i_{1}}{2^{N}}, \ldots, \frac{i_{n}}{2^{N}}\right):\left(i_{1}, \ldots, i_{n}\right) \in \mathbb{N}^{n}\right\} \cap[0,1]^{n}
$$

For any $x \in G$ and $i \leqslant n$, let $x_{i}^{ \pm}$denote the point $x \pm 2^{-N} e_{i}$; that is the neighbouring point of $x$ in $G$ in the positive/negative direction of the $i$ th coordinate. For any $x \in G$ and $i \leqslant n$ fix 
$\lambda_{x, i}^{+} \in\{-1,0,1\}$ and $\lambda_{x, i}^{-} \in\{-1,0,1\}$, such that

$$
\begin{aligned}
\lambda_{x, i}^{+}=0 & \Longrightarrow\left|\frac{\partial h}{\partial x_{i}}\left(x+2^{-(N+1)} e_{i}\right)\right|<\frac{3 \varepsilon}{4}, \\
\lambda_{x, i}^{+}=-1 & \Longrightarrow \frac{\partial h}{\partial x_{i}}\left(x+2^{-(N+1)} e_{i}\right)<-\frac{\varepsilon}{2}, \\
\lambda_{x, i}^{+}=1 & \Longrightarrow \frac{\partial h}{\partial x_{i}}\left(x+2^{-(N+1)} e_{i}\right)>\frac{\varepsilon}{2}, \\
\lambda_{x, i}^{-}=0 & \Longrightarrow\left|\frac{\partial h}{\partial x_{i}}\left(x-2^{-(N+1)} e_{i}\right)\right|<\frac{3 \varepsilon}{4}, \\
\lambda_{x, i}^{-}=-1 & \Longrightarrow \frac{\partial h}{\partial x_{i}}\left(x-2^{-(N+1)} e_{i}\right)>\frac{\varepsilon}{2}, \\
\lambda_{x, i}^{-}=1 & \Longrightarrow \frac{\partial h}{\partial x_{i}}\left(x-2^{-(N+1)} e_{i}\right)<-\frac{\varepsilon}{2} .
\end{aligned}
$$

Notice that if $\lambda_{x, i}^{+}=-1$ then for all $y \in\left[x, x_{i}^{+}\right]$,

and therefore, by Lemma 2.3,

$$
\frac{\partial h}{\partial x_{i}}(y)<-\frac{\varepsilon}{4}
$$

$$
h(x)>h\left(x_{i}^{+}\right) .
$$

Similarly, when $\lambda_{x, i}^{-}=-1$, we obtain

$$
\frac{\partial h}{\partial x_{i}}(y)>\frac{\varepsilon}{4}
$$

for all $y \in\left[x_{i}^{-}, x\right]$, and so

$$
h(x)>h\left(x_{i}^{-}\right) .
$$

Furthermore, notice that, by (2),

$$
\text { if } \lambda_{x, i}^{+} \in\{0,1\} \text { and } \lambda_{x, i}^{-} \in\{0,1\} \text {, then }\left|\frac{\partial h}{\partial x_{i}}(x)\right|<\varepsilon .
$$

By (3), we can find $x_{0} \in G$ such that $\left|h\left(x_{0}\right)-h(\xi)\right|<s$; whence, in view of (1), $h(x)>h\left(x_{0}\right)$ for all $x \in \partial[0,1]^{n}$. If there exists $i$ such that $\lambda_{x_{0}, i}^{+}=-1$ (respectively, $\lambda_{x_{0}, i}^{-}=-1$ ), set $x_{1}=\left(x_{0}\right)_{i}^{+}$ (respectively, $x_{1}=\left(x_{0}\right)_{i}^{-}$), for which $h\left(x_{0}\right)>h\left(x_{1}\right)$. It follows from (4) that if we continue this construction, then we will never visit the same point twice and never reach a point in $\partial[0,1]^{n} \cap G$. Therefore, the construction has to stop eventually and we reach a point $x_{m} \in(0,1)^{n} \cap G$ for which both $\lambda_{x_{m}, i}^{+} \neq-1$ and $\lambda_{x_{m}, i}^{-} \neq-1$ for all $1 \leqslant i \leqslant n$. By (6) we have that $\left\|\nabla h\left(x_{m}\right)\right\|<\varepsilon$.

\section{Uniqueness and continuity}

The results in this section are taken from [18], with the proofs adjusted in parts in order to ensure they are fully constructive.

Situation. Let $U \subseteq \mathbb{R}^{n}$ and $V \subseteq \mathbb{R}^{m}$ be open neighbourhoods of $a \in \mathbb{R}^{n}$ and $b \in \mathbb{R}^{m}$, respectively, where $m, n \geqslant 1$. We denote the coordinates on $\mathbb{R}^{n}$ and $\mathbb{R}^{m}$ by $x=\left(x_{1}, \ldots, x_{n}\right)$ and $y=\left(y_{1}, \ldots, y_{m}\right)$, respectively, and endow $\mathbb{R}^{n} \times \mathbb{R}^{m}$ with the norm $\|(x, y)\|=\|x\|+\|y\|$. The Jacobian of a partially differentiable function $F: U \times V \rightarrow \mathbb{R}^{m}$ at $(x, y) \in U \times V$ is written 
as

$$
D F(x, y)=\left(\frac{\partial F}{\partial x}(x, y), \frac{\partial F}{\partial y}(x, y)\right), \quad \frac{\partial F}{\partial x}(x, y) \in \mathbb{R}^{m \times n}, \quad \frac{\partial F}{\partial y}(x, y) \in \mathbb{R}^{m \times m}
$$

Finally, let $F: U \times V \rightarrow \mathbb{R}^{m}$ be a continuously differentiable function such that $(\partial F / \partial y)(a, b)$ is invertible; in particular $\nu>0$ where

$$
\nu=\left\|\frac{\partial F}{\partial y}(a, b)^{-1}\right\|
$$

LEmma 3.1. For every $\lambda \in] 1,+\infty\left[\right.$ there are compact neighbourhoods $U_{\lambda} \subseteq U$ and $V_{\lambda} \subseteq V$ of $a$ and $b$, respectively, such that for all $x \in U_{\lambda}$ and $y, y^{\prime} \in V_{\lambda}$ :

$$
\left\|y-y^{\prime}\right\| \leqslant \lambda \cdot \nu \cdot\left\|F(x, y)-F\left(x, y^{\prime}\right)\right\| \text {. }
$$

Proof. By replacing $F$ with $(\partial F / \partial y)(a, b)^{-1} \cdot F$, we may assume that $(\partial F / \partial y)(a, b)$ is the identity matrix and therefore that $\nu=1$. Now consider

$$
G: U \times V \rightarrow \mathbb{R}^{m}, \quad(x, y) \mapsto y-F(x, y) .
$$

Since $G$ is continuously differentiable with $(\partial G / \partial y)(a, b)=0$, there are compact neighbourhoods $U_{\lambda} \subseteq U$ and $V_{\lambda} \subseteq V$ of $a$ and $b$, respectively, such that $V_{\lambda}$ is convex and

$$
\left\|\frac{\partial G}{\partial y}(x, y)\right\| \leqslant 1-1 / \lambda
$$

for all $(x, y) \in U_{\lambda} \times V_{\lambda}$. Then, for all $x \in U_{\lambda}$ and $y, y^{\prime} \in V_{\lambda}$, we have

$$
\begin{aligned}
\left\|y-y^{\prime}\right\| & \leqslant\left\|(y-G(x, y))-\left(y^{\prime}-G\left(x, y^{\prime}\right)\right)\right\|+\left\|G(x, y)-G\left(x, y^{\prime}\right)\right\| \\
& \leqslant\left\|F(x, y)-F\left(x, y^{\prime}\right)\right\|+(1-1 / \lambda) \cdot\left\|y-y^{\prime}\right\|
\end{aligned}
$$

by (8) and Corollary 2.2; whence (7) holds with $\nu=1$.

Throughout the following, $\lambda \in] 1,+\infty\left[\right.$ is arbitrary and $U_{\lambda}, V_{\lambda}$ are as in Lemma 3.1.

Equation (7) implies, for fixed $x \in U_{\lambda}$, that $y \in V_{\lambda}$ and $y^{\prime} \in V_{\lambda}$ lie close together whenever $F$ is small at $(x, y)$ and $\left(x, y^{\prime}\right)$. Therefore $(7)$ can be seen as a quantitative way to express that any $y$ with $F(x, y)=0$ is uniquely determined by $x$.

Corollary 3.2. For each $x \in U_{\lambda}$ there is at most one $y \in V_{\lambda}$ with $F(x, y)=0$.

In other words, we have for all $x \in U_{\lambda}$ and $y, y^{\prime} \in V_{\lambda}$ that

$$
F(x, y)=0 \wedge F\left(x, y^{\prime}\right)=0 \Rightarrow y=y^{\prime}
$$

ThEOREM 3.3. Every function $f: U_{\lambda} \rightarrow V_{\lambda}$ with $F(x, f(x))=0$ for all $x \in U_{\lambda}$ is continuous.

Proof. Consider $\varepsilon>0$ arbitrary. Since $F$ is uniformly continuous on the compact set $U_{\lambda} \times V_{\lambda}$, there exists $\delta>0$ such that

$$
\left\|F(x, y)-F\left(x^{\prime}, y^{\prime}\right)\right\| \leqslant(\lambda \cdot \nu)^{-1} \cdot \varepsilon
$$

whenever $(x, y),\left(x^{\prime}, y^{\prime}\right) \in U_{\lambda} \times V_{\lambda}$ are such that $\left\|x-x^{\prime}\right\|+\left\|y-y^{\prime}\right\|<\delta$. In particular,

$$
\left\|F\left(x, f\left(x^{\prime}\right)\right)\right\| \leqslant(\lambda \cdot \nu)^{-1} \cdot \varepsilon
$$


for all $x, x^{\prime} \in U_{\lambda}$ with $\left\|x-x^{\prime}\right\|<\delta$ (recall that $F\left(x^{\prime}, f\left(x^{\prime}\right)\right)=0$ ). Using this and (7) we obtain

$$
\begin{aligned}
\left\|f(x)-f\left(x^{\prime}\right)\right\| & \leqslant \lambda \cdot \nu \cdot\left\|F(x, f(x))-F\left(x, f\left(x^{\prime}\right)\right)\right\| \\
& =\lambda \cdot \nu \cdot\left\|F\left(x, f\left(x^{\prime}\right)\right)\right\| \\
& \leqslant \varepsilon
\end{aligned}
$$

for all $x, x^{\prime} \in U_{\lambda}$ with $\left\|x-x^{\prime}\right\|<\delta$. Hence $f$ is (uniformly) continuous.

The idea of the proof can be explained as follows. If $x$ and $x^{\prime}$ are close, then $F\left(x, f\left(x^{\prime}\right)\right)$ is close to $F\left(x^{\prime}, f\left(x^{\prime}\right)\right)=0$, and therefore close to $F(x, f(x))=0$; equation (7) now implies that $f(x)$ and $f\left(x^{\prime}\right)$ are close.

Following the standard argument, one can now easily show that if $f$ satisfies the conditions of Theorem 3.3, then it is differentiable in the interior of $U_{\lambda}$ with a uniformly continuous derivative such that

$$
D f(x)=-\frac{\partial F}{\partial y}(x, f(x))^{-1} \cdot \frac{\partial F}{\partial x}(x, f(x)) .
$$

Note that the quantitative version (7) of uniqueness was sufficient to prove the continuity of the implicit function $f$; therefore the continuity of $f$ depends only on the differentiability of the defining equation.

We further observe that the invertibility of $(\partial F / \partial y)(x, y)$ for fixed $x$ and $y$ is even necessary for the validity of (7) for all $y^{\prime}$ close to $y$.

Remark 3.4. Let $X$ be an open subset of $\mathbb{R}^{k}$ and let $h: X \rightarrow \mathbb{R}^{k}$ be a function which is differentiable at $x_{0} \in X$. If there is $\eta>0$ with

$$
\eta \cdot\left\|x-x_{0}\right\| \leqslant\left\|h(x)-h\left(x_{0}\right)\right\|
$$

for all $x \in X$, then $D h\left(x_{0}\right)$ is an invertible matrix.

Proof. If $0<\varepsilon<\eta$, then there is $\delta>0$ such that

$$
\left\|h\left(x_{0}+\xi\right)-h\left(x_{0}\right)\right\|-\left\|D h\left(x_{0}\right) \cdot \xi\right\| \leqslant \varepsilon \cdot\|\xi\|
$$

for all $\xi \in \mathbb{R}^{k}$ with $\|\xi\| \leqslant \delta$ and $x_{0}+\xi \in X$. Hence

$$
(\eta-\varepsilon) \cdot\|\xi\| \leqslant\left\|D h\left(x_{0}\right) \cdot \xi\right\|
$$

and so $D h\left(x_{0}\right)$ is an invertible matrix.

\section{Uniqueness and existence}

In this section we present an alternative approach to the existence of the implicit function which, like the proof of continuity, is based on the quantitative version (7) of uniqueness; again this requires the involvement of the partial derivative of the given equation. To start, we need to make the above discussion of Lemma 3.1 more precise.

A function $H: S \rightarrow \mathbb{R}$ on a metric space $S$ with $H \geqslant 0$ has uniformly at most one root if

$$
\forall \delta>0 \exists \varepsilon>0 \forall y, y^{\prime} \in S\left[H(y)<\varepsilon \wedge H\left(y^{\prime}\right)<\varepsilon \Rightarrow d\left(y, y^{\prime}\right)<\delta\right],
$$

or, equivalently (notice that, for $\varepsilon>0$ and $r, s \in \mathbb{R}$, if $\neg(r<\varepsilon \wedge s<\varepsilon$ ), then $r>\varepsilon / 2 \vee s>\varepsilon / 2$.), if

$$
\forall \delta>0 \exists \varepsilon>0 \forall y, y^{\prime} \in S\left[d\left(y, y^{\prime}\right) \geqslant \delta \Rightarrow H(y) \geqslant \varepsilon \vee H\left(y^{\prime}\right) \geqslant \varepsilon\right] .
$$

If $H$ has uniformly at most one root, then the root, if it exists at all, is uniquely determined:

$$
\forall y, y^{\prime} \in S\left[H(y)=0 \wedge H\left(y^{\prime}\right)=0 \Rightarrow y=y^{\prime}\right] .
$$


The next lemma is an immediate consequence of Lemma 3.1 above.

LEMma 4.1. For each $x \in U_{\lambda}$ the function

$$
H: V_{\lambda} \rightarrow \mathbb{R}, y \mapsto\|F(x, y)\|
$$

has uniformly at most one root.

Why do we bother at all about uniform uniqueness when we already have ordinary uniqueness (Corollary 3.2)? We want to use the following instrument, for whose impressive history we refer to $[\mathbf{1 7}, \mathbf{1 9}]$. It is valid also in Bishop-style constructive mathematics without choice [19].

ThEOREm 4.2. Let $S$ be a complete metric space and let $H: S \rightarrow \mathbb{R}$ be a uniformly continuous function. If inf $H=0$ and $H$ has uniformly at most one root, then there is $y \in S$ with $H(y)=0$.

Clearly, the root $y$ whose existence is asserted in Theorem 4.2 is uniquely determined. To be able to apply Theorem 4.2, we still need to verify the existence of approximate roots, as follows.

Hereafter we assume that $F(a, b)=0$ (an assumption that has not been used so far).

Lemma 4.3. There are compact neighbourhoods $U_{\lambda}^{0} \subseteq U_{\lambda}$ and $V_{\lambda}^{0} \subseteq V_{\lambda}$ of $a$ and $b$, respectively, such that for $x \in U_{\lambda}^{0}$,

$$
\inf _{y \in V_{\lambda}^{0}}\|F(x, y)\|=0
$$

Proof. We may assume that $(a, b)=(0,0)$. Setting $\left(x, y^{\prime}\right)=(0,0)$ in $(7)$, we obtain

$$
\|y\| \leqslant \lambda \cdot \nu \cdot\|F(0, y)\|
$$

for all $y \in U_{\lambda}$, since $F(0,0)=0$. We can now find $r, s>0$, such that

$$
U_{\lambda}^{0}=[-r,+r]^{n}, \quad V_{\lambda}^{0}=[-s,+s]^{m}
$$

lie completely within $U_{\lambda}$ and $V_{\lambda}$, respectively. By choosing $r, s$ small enough, we may assume that

$$
\left\|\frac{\partial F}{\partial y}(x, y)^{-1}\right\|>\frac{\nu}{2}
$$

for all $(x, y) \in U_{\lambda}^{0} \times V_{\lambda}^{0}$. Since $F$ is uniformly continuous on the compact set $U_{\lambda}^{0} \times V_{\lambda}^{0}$, by making $r$ sufficiently small, we may further assume that

$$
\lambda \cdot \nu \cdot\left\|F(x, y)-F\left(x^{\prime}, y\right)\right\| \leqslant s / 3
$$

for all $(x, y),\left(x^{\prime}, y\right) \in U_{\lambda}^{0} \times V_{\lambda}^{0}$. If we now substitute $x^{\prime}=0$, we obtain

$$
\lambda \cdot \nu \cdot\|F(x, y)-F(0, y)\| \leqslant s / 3
$$

for all $(x, y) \in U_{\lambda}^{0} \times V_{\lambda}^{0}$; if we also substitute $y=0$, we obtain

$$
\lambda \cdot \nu \cdot\|F(x, 0)\| \leqslant s / 3
$$

for all $x \in U_{\lambda}^{0}$. (If we were only interested in obtaining (12), it would suffice to point out that $F(\cdot, 0)$ is continuous at 0 and that $F(0,0)=0$.) Equations $(9)$ and (11) imply that

$$
2 s / 3 \leqslant \lambda \cdot \nu \cdot\|F(x, y)\|
$$


for all $x \in U_{\lambda}^{0}$ and $y \in \partial V_{\lambda}^{0}$, that is $\|y\|=s$. Now consider $x \in U_{\lambda}^{0}$ arbitrary, but fixed. The function

$$
h: V \rightarrow \mathbb{R}, y \mapsto\|F(x, y)\|^{2}
$$

is continuously differentiable, and by (12) and (13) we have

$$
\lambda^{2} \cdot \nu^{2} \cdot h(0)+s^{2} / 3 \leqslant \lambda^{2} \cdot \nu^{2} \cdot h(y)
$$

for all $y \in \partial V_{\lambda}^{0}$; whence, by virtue of Lemma 2.4,

$$
\inf _{y \in V_{\lambda}^{0}}\|\nabla h(y)\|=0 .
$$

The derivative of $h$ can easily be computed as

$$
\nabla h(y)=2 \cdot F(x, y) \cdot \frac{\partial F}{\partial y}(x, y)
$$

By multiplying with $(\partial F / \partial y)(x, y)^{-1}$ and in view of (10) we thus have

$$
\inf _{y \in V_{\lambda}^{0}}\|F(x, y)\|=0
$$

as required.

Now let $U_{\lambda}^{0}$ and $V_{\lambda}^{0}$ be as in Lemma 4.3. By Lemma 4.1, Theorem 4.2 and Lemma 4.3, for every $x \in U_{\lambda}^{0}$ there exists a unique $y \in V_{\lambda}^{0}$ with $F(x, y)=0$. Hence we have achieved the desired result as follows.

TheOREm 4.4. There is a function $f: U_{\lambda}^{0} \rightarrow V_{\lambda}^{0}$ with $F(x, f(x))=0$ for every $x \in U_{\lambda}^{0}$.

Needless to say, there is exactly one $f$ as in Theorem 4.4, which by Theorem 3.3 is continuous.

\section{Implicit functions and unique existence with parameters}

We sketch how Theorem 4.4 can also be deduced from a parametrised variant of Theorem 4.2. In this section, let $S, T$ be metric spaces and let $F: T \times S \rightarrow \mathbb{R}$ be a function with $F \geqslant 0$. We will suppose, in addition, that $F$ satisfies, with $\delta, \varepsilon>0, x, x^{\prime} \in T$, and $y, y^{\prime} \in S$, the condition

$$
\forall \delta \forall x \exists \varepsilon \forall x^{\prime} \forall y, y^{\prime}\left[d\left(x, x^{\prime}\right)<\varepsilon \wedge F(x, y)<\varepsilon \wedge F\left(x^{\prime}, y^{\prime}\right)<\varepsilon \Rightarrow d\left(y, y^{\prime}\right)<\delta\right]
$$

or even its uniform variant

$$
\forall \delta \exists \varepsilon \forall x \forall x^{\prime} \forall y, y^{\prime}\left[d\left(x, x^{\prime}\right)<\varepsilon \wedge F(x, y)<\varepsilon \wedge F\left(x^{\prime}, y^{\prime}\right)<\varepsilon \Rightarrow d\left(y, y^{\prime}\right)<\delta\right] .
$$

Before any talk of existence, note that $F(x, y)=0$ defines a pointwise continuous (respectively, uniformly continuous) partial function $x \mapsto y$ whenever $F$ satisfies (14) (respectively (15)). In other words, uniqueness with parameters implies continuity. (To see this, consider equations (14) and (15) for $x=x^{\prime}$ and under the precondition that $F(x, y)=0$ and $F\left(x^{\prime}, y^{\prime}\right)=0$.)

Already, the case $x=x^{\prime}$ of (14) says that $F(x, \cdot)$ has uniformly at most one root for every $x \in T$. Hence the partial function $x \mapsto y$ defined by $F(x, y)=0$ is total whenever $F(x, \cdot)$ satisfies the other hypotheses of Theorem 4.2 for every $x \in T$. Here we use the following.

FACT. Let $S$ be complete and let $F(x, \cdot)$ be uniformly continuous with inf $F(x, \cdot)=0$ for each $x \in T$. If $F$ satisfies (14) or even (15), then there is a pointwise or even uniformly continuous function $f: T \rightarrow S$ with $F(x, f(x))=0$ for all $x \in T$. 
We again refer to $[\mathbf{1 7}, \mathbf{1 9}]$ for the history of this, including references.

To see that this subsumes the implicit function theorem, we return to the situation and the notation of the preceding sections. In view of Lemma 4.3 we only have to show that (7), from Lemma 3.1, results in (15) with $U_{\lambda}^{0}, V_{\lambda}^{0}$ and $\|F\|$ in place of $T, S$ and $F$, respectively (and, of course, with the metrics induced by the norms).

To this end, let $\delta>0$ be given; set $\rho=\delta /(3 \lambda \nu)$. Since $F$ is uniformly continuous on the compact set $U_{\lambda}^{0} \times V_{\lambda}^{0}$, there is $\varepsilon>0$ with $\varepsilon \leqslant \rho$ such that

$$
\left\|F\left(x, y^{\prime}\right)-F\left(x^{\prime}, y^{\prime}\right)\right\|<\rho
$$

whenever $x, x^{\prime} \in U_{\lambda}^{0}$ and $y, y^{\prime} \in V_{\lambda}^{0}$ with $\left\|x-x^{\prime}\right\|<\varepsilon$. (Recall our particular choice of the norm on the product: $\|(x, y)\|=\|x\|+\|y\|$.) With such $x, x^{\prime}$ and $y, y^{\prime}$, if

$$
\|F(x, y)\|<\varepsilon \text { and }\left\|F\left(x^{\prime}, y^{\prime}\right)\right\|<\varepsilon \text {, }
$$

then by (7) we have

$$
\begin{aligned}
\left\|y-y^{\prime}\right\| & \leqslant \lambda \cdot \nu \cdot\left\|F(x, y)-F\left(x, y^{\prime}\right)\right\| \\
& \leqslant \lambda \cdot \nu \cdot\left(\|F(x, y)\|+\left\|F\left(x, y^{\prime}\right)-F\left(x^{\prime}, y^{\prime}\right)\right\|+\left\|F\left(x^{\prime}, y^{\prime}\right)\right\|\right) \\
& <\lambda \cdot \nu \cdot(\varepsilon+\rho+\varepsilon) \\
& \leqslant 3 \cdot \lambda \cdot \nu \cdot \rho \\
& =\delta
\end{aligned}
$$

as required in (15).

Acknowledgements. A lecture on implicit functions that the second author gave at the University of Bern made him first think of the subject matter present in this paper. Next, D. S. Bridges pointed the authors to [6]; O. Forster and H. Kalf gave useful hints. The first author would like to thank the Department of Mathematics and Statistics at the University of Canterbury for supporting him as an outgoing postdoctoral fellow. During the preparation of this paper the second author was holding a Feodor Lynen Research Fellowship for Experienced Researchers granted by the Alexander von Humboldt Foundation from sources of the German Federal Ministry of Education and Research; he is grateful to A. Cantini and G. Sambin for their hospitality in Florence and Padua, respectively. Both authors would also like to thank Matthew Hendtlass for suggesting many changes that improved the presentation of this paper.

\section{References}

1. P. ACzel, 'The type theoretic interpretation of constructive set theory', Logic colloquium '77 (eds A. Macintyre, L. Pacholski and J. Paris; North-Holland, Amsterdam, 1978) 55-66.

2. P. Aczel and M. Rathjen, 'Notes on constructive set theory', Institut Mittag-Leffler Preprint No. 40, $2000 / 01$

3. J. Berger, D. Bridges and P. Schuster, 'The fan theorem and unique existence of maxima', J. Symbolic Logic 71 (2006) 713-720.

4. E. Bishop, Foundations of constructive analysis (McGraw-Hill, New York, 1967).

5. E. Bishop and D. Bridges, Constructive analysis (Springer, Berlin, 1985).

6. D. Bridges, C. Calude, B. Pavlov and D. Ştefănescu, 'The constructive implicit function theorem and applications in mechanics', Chaos Solitons Fractals 10 (1999) 927-934.

7. D. Bridges and F. Richman, Varieties of constructive mathematics (Cambridge University Press, Cambridge, 1987).

8. D. BRIDGES and L. Vîţ̆ Ă, Techniques of constructive analysis (Springer, New York, 2006).

9. H. Diener and P. Schuster, 'Uniqueness, continuity, and existence of implicit functions in constructive analysis', Sixth Internat. Conf. on Computability and Complexity in Analysis 2009 (eds A. Bauer, P. Hertling and Ker-I Ko; Schloß Dagstuhl Leibniz-Zentrum für Informatik, Dagstuhl, 2009), http://drops.dagstuhl.de/opus/volltexte/2009/2265.

10. O. Forster, Analysis 2: differentialrechnung im $\mathbb{R}^{n}$, gewöhnliche differentialgleichungen, 6th edn (Vieweg, Wiesbaden, 2005). 
11. S. G. Krantz and H. R. PARKs, The implicit function theorem. History, theory, and applications (Birkhäuser, Boston, 2002).

12. T. H. MCNichold, 'A uniformly computable implicit function theorem', MLQ Math. Log. Q. 54 (2008) $272-279$.

13. M. Rathjen, 'Choice principles in constructive and classical set theories', Logic colloquium 'O2. Proceedings, Münster, 2002, Lecture Notes in Logic 27 (eds Z. Chatzidakis, P. Koepke and W. Pohlers; Assoc. Symbol. Logic, La Jolla, 2006) 299-326.

14. F. Richman, 'Intuitionism as generalization', Philos. Math. (3) 5 (1990) 124-128.

15. F. Richman, 'The fundamental theorem of algebra: a constructive development without choice', Pacific J. Math. 196 (2000) 213-230.

16. P. Schuster, 'What is continuity, constructively?', J. UCS 11 (2005) 2076-2085.

17. P. Schuster, 'Unique solutions', Math. Log. Q. 52 (2006) 534-539; Corrigendum: Math. Log. Q. 53 (2007) 214.

18. P. Schuster, 'Zur Eindeutigkeit impliziter Funktionen', Preprint, Universitá di Firenze, 2009.

19. P. Schuster, 'Problems, solutions, and completions', J. Log. Algebr. Program. 79 (2010) 84-91.

20. K. Weinrauch, Computable analysis. An introduction (Springer, Berlin, 2000).

21. M. Ziegler, 'Effectively open real functions', J. Complexity 22 (2006) 827-849.

\section{H. Diener}

Universitat Siegen

Fachbereich 6: Mathematik

Walter-Flex-Str. 3

57072 Siegen

Germany

diener@math.uni-siegen.de

\section{P. Schuster}

Pure Mathematics

University of Leeds

LS2 9JT

U.K.

pschust@maths.leeds.ac.uk 